\title{
Crystal structure of 2,3-O-dimethyl-5,6-O-isopropylidene-L-ascorbic acid, $\mathrm{C}_{11} \mathrm{H}_{16} \mathrm{O}_{6}$
}

\author{
A. Ries, J. Sander and K. Hegetschweiler* \\ Universität des Saarlandes, Anorganische Chemie, Postfach 151150, D-66041 Saarbrücken, Germany
}

Received May 8, 2001, CCDC-No. 1267/657

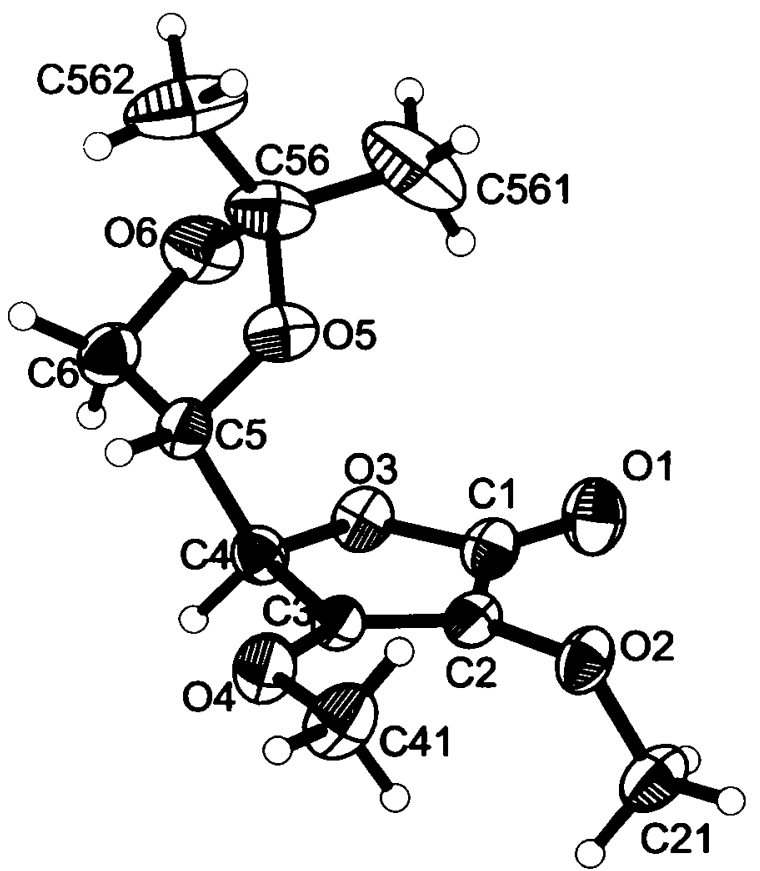

\footnotetext{
Abstract

$\mathrm{C}_{11} \mathrm{H}_{16} \mathrm{O}_{6}$, trigonal, $P 3_{2}$ (No. 145), $a=12.043(2) \AA$ $c=7.666(2) \AA, V=962.9 \AA^{3}, Z=3, R_{\mathrm{gt}}(F)=0.037$, $w R_{\text {ref }}\left(F^{2}\right)=0.122, T=293 \mathrm{~K}$.
}

\section{Source of material}

2,3-O-Dimethyl-5,6- $O$-isopropylidene-L-ascorbic acid was synthesized as described elsewhere [1]. Crystals were grown by slow evaporation of a solution of the title compound in isopropanol/ water $(1: 1)$.

\section{Experimental details}

All hydrogen atoms were placed at calculated sites using a riding model. Isotropic displacement parameters were used which were fixed to a value corresponding to the $U_{\mathrm{eq}}$ value of the bonded atom multiplied with a factor of 1.2. In the figure, the displacement ellipsoids are drawn at the $50 \%$ probability level. The hydrogen atoms are shown in arbitrary size.

\section{Discussion}

Due to the well established importance in food science and nutrition [2], the chemistry of L-ascorbic acid has intensively been studied [1]. The four hydroxy groups of this compound allow selective alkylation, and a variety of such chiral derivatives have been prepared. However, only a few crystal structures of such compounds have been reported [3-6]. In the title compound, a vitamin $\mathrm{C}$ derivative, the four hydroxy groups of $\mathrm{L}$-ascorbic acid are all alkylated. The molecule contains two five membered rings with different conformations. The furanone ring is practically planar: the deviation of the mean plane is less than $0.04 \AA$ for the five ring atoms, whereas the 1,3-dioxolane ring has a puckered conformation corresponding to an envelope.

Table 1. Data collection and handling.

\section{Crystal:}

Wavelength:

$\mu:$

Diffractometer, scan mode:

$2 \theta_{\max }$ :

$N(h k l)_{\text {measured, }}, N(h k l)_{\text {unique: }}$

Criterion for $I_{\mathrm{obs}}, N(h k l)_{\mathrm{gt}}$ :

$N(\text { param })_{\text {refined: }}$

Programs: colourless plate, size $0.2 \times 0.3 \times 0.4 \mathrm{~mm}$

Mo $K_{\alpha}$ radiation $(0.71073 \AA)$

$1.38 \mathrm{~cm}^{-1}$

Stoe IPDS, $\phi$

$48.2^{\circ}$

6056,1897

$I_{\text {obs }}>2 \sigma\left(I_{\text {obs }}\right), 1683$

156

SHELXS-97 [7], SHELXL-97 [8]
Table 2. Atomic coordinates and displacement parameters (in $\AA^{2}$ ).

\begin{tabular}{lllrrl}
\hline Atom & Site & \multicolumn{1}{l}{$x$} & \multicolumn{1}{l}{$y$} & $U_{\text {iso }}$ \\
\hline H(4) & $3 a$ & 0.4836 & 0.1562 & -0.0775 & 0.061 \\
H(21A) & $3 a$ & 0.1009 & -0.0762 & 0.4759 & 0.109 \\
H(21B) & $3 a$ & 0.2481 & 0.0224 & 0.4886 & 0.109 \\
H(21C) & $3 a$ & 0.1984 & -0.0960 & 0.3636 & 0.109 \\
H(5) & $3 a$ & 0.3877 & 0.2242 & -0.2919 & 0.063 \\
H(6A) & $3 a$ & 0.6270 & 0.3708 & -0.2006 & 0.077 \\
H(6B) & $3 a$ & 0.5663 & 0.4034 & -0.3614 & 0.077 \\
H(41A) & $3 a$ & 0.0625 & -0.1474 & -0.1702 & 0.121 \\
H(41B) & $3 a$ & 0.0548 & -0.0487 & -0.0445 & 0.121 \\
H(41C) & $3 a$ & 0.1005 & -0.1413 & 0.0266 & 0.121 \\
H(56A) & $3 a$ & 0.3401 & 0.5156 & 0.0363 & 0.201 \\
H(56B) & $3 a$ & 0.4867 & 0.6175 & 0.0325 & 0.201 \\
H(56C) & $3 a$ & 0.4392 & 0.4851 & 0.1247 & 0.201 \\
H(56D) & $3 a$ & 0.3234 & 0.5052 & -0.3028 & 0.211 \\
H(56E) & $3 a$ & 0.4189 & 0.4770 & -0.4062 & 0.211 \\
H(56F) & $3 a$ & 0.4682 & 0.6112 & -0.3182 & 0.211 \\
\hline
\end{tabular}


Table 3. Atomic coordinates and displacement parameters (in $\AA^{2}$ ).

\begin{tabular}{|c|c|c|c|c|c|c|c|c|c|c|}
\hline Atom & Site & $x$ & $y$ & : & $U_{11}$ & $U_{22}$ & $U_{33}$ & $U_{12}$ & $U_{13}$ & $U_{23}$ \\
\hline$O(3)$ & $3 a$ & $0.4815(2)$ & $0.2681(2)$ & $0.1133(2)$ & $0.0436(9)$ & $0.063(1)$ & $0.052(1)$ & $0.0207(8)$ & $-0.0072(8)$ & $0.0000(8)$ \\
\hline$O(2)$ & $3 a$ & $0.1683(2)$ & $0.0404(2)$ & $0.2732(3)$ & $0.048(1)$ & $0.080(1)$ & $0.055(1)$ & $0.0326(9)$ & $0.0105(8)$ & $0.0162(9)$ \\
\hline$O(5)$ & $3 a$ & $0.3571(2)$ & $0.3356(2)$ & $-0.1334(3)$ & $0.0464(9)$ & $0.055(1)$ & $0.083(2)$ & $0.0229(8)$ & $-0.0069(9)$ & $0.0075(9)$ \\
\hline$O(6)$ & $3 a$ & $0.5668(2)$ & $0.4927(2)$ & $-0.1436(4)$ & $0.053(1)$ & $0.056(1)$ & $0.114(2)$ & $0.0158(8)$ & $-0.002(1)$ & $-0.004(1)$ \\
\hline$O(4)$ & $3 a$ & $0.2326(2)$ & $0.0021(2)$ & $-0.1143(3)$ & $0.068(1)$ & $0.054(1)$ & $0.061(1)$ & $0.0110(9)$ & $0.003(1)$ & $-0.0079(9)$ \\
\hline $\mathrm{C}(2)$ & $3 a$ & $0.2766(2)$ & $0.1058(2)$ & $0.1739(4)$ & $0.042(1)$ & $0.057(1)$ & $0.051(2)$ & $0.026(1)$ & $0.003(1)$ & $0.010(1)$ \\
\hline$C(3)$ & $3 a$ & $0.2995(2)$ & $0.0875(2)$ & $0.0090(3)$ & $0.049(1)$ & $0.048(1)$ & $0.044(2)$ & $0.023(1)$ & $0.001(1)$ & $0.003(1)$ \\
\hline$C(4)$ & $3 a$ & $0.4303(2)$ & $0.1930(2)$ & $-0.0430(4)$ & $0.045(1)$ & $0.051(1)$ & $0.056(2)$ & $0.023(1)$ & $0.003(1)$ & $-0.004(1)$ \\
\hline$C(21)$ & $3 a$ & $0.1799(3)$ & $-0.0335(3)$ & $0.4118(5)$ & $0.064(2)$ & $0.080(2)$ & $0.071(2)$ & $0.033(2)$ & $0.015(1)$ & $0.028(2)$ \\
\hline$C(5)$ & $3 a$ & $0.4282(2)$ & $0.2769(2)$ & $-0.1883(4)$ & $0.050(1)$ & $0.052(1)$ & $0.046(2)$ & $0.019(1)$ & $0.003(1)$ & $-0.001(1)$ \\
\hline$C(1)$ & $3 a$ & $0.3876(2)$ & $0.2224(3)$ & $0.2380(4)$ & $0.051(1)$ & $0.062(2)$ & $0.042(2)$ & $0.028(1)$ & $-0.008(1)$ & $0.001(1)$ \\
\hline$C(6)$ & $3 a$ & $0.5596(3)$ & $0.3877(3)$ & $-0.2367(4)$ & $0.061(2)$ & $0.054(2)$ & $0.064(2)$ & $0.017(1)$ & $0.009(1)$ & $0.006(1)$ \\
\hline$C(56)$ & $3 a$ & $0.4390(3)$ & $0.4715(3)$ & $-0.1416(6)$ & $0.066(2)$ & $0.052(2)$ & $0.128(3)$ & $0.028(1)$ & $-0.010(2)$ & $0.002(2)$ \\
\hline$C(41)$ & $3 a$ & $0.1016(3)$ & $-0.0917(3)$ & $-0.0721(5)$ & $0.066(2)$ & $0.066(2)$ & $0.073(3)$ & $0.006(2)$ & $-0.008(2)$ & $0.000(2)$ \\
\hline$C(562)$ & $3 a$ & $0.4097(5)$ & $0.5207(5)$ & $-0.307(1)$ & $0.119(4)$ & $0.088(3)$ & $0.210(7)$ & $0.048(3)$ & $-0.028(4)$ & $0.052(4)$ \\
\hline
\end{tabular}

Acknowledgments. Financial support by Vifor Inc. (St. Gallen, Switzerland) is gratefully acknowledged. We thank Prof. Dr. M. Veith for providing the diffraction facility and Dr. V. Huch for the collection of the data set.

\section{References}

1. Dallacker, F.; Sanders, J.: Darstellung und Eigenschaften der $O^{2}, O^{3}$ Ethandiyl- und der $O^{2}, O^{3}$-Dibenzyl-L-ascorbinsäuren. Chem. Z. 109 (1985) 197-202.

2. Maeda, N.; Hagihara, H.; Nakata, Y.; Hiller, S.; Wilder, J.; Reddick, R.: Aortic wall damage in mice unable to synthesize ascorbic acid. Proc. Nat. Acad. Sci. U.S.A. 97 (2000) 841-846.

3. McClelland, B. W.: Crystal and Molecular Structure of Barium 2-O-Sulfonato-L-ascorbate Dihydrate. Acta Crystallogr. B30 (1974) 178-186.

4. Jemow, J.; Blount, J.; Oliveto, E.; Perrotta, A.; Rosen, P.; Toome, V.: Structural determination of ascorbic acid 2- $O$-phosphate formed via acid hydrolysis of an ascorbic acid 3-O-phosphinate. Tetrahedron 35 (1979) 1483-1486.
5. Nomura, H.; Nakamachi, H.; Wada, Y.: X-ray analysis of L-Ascorbic Acid 2-O-Phosphate. Chem. Pharm. Bull. 30 (1982) 1024-1029.

6. Mandai, T.; Yoneyama, M.; Sakai, S.; Muto, N.; Yamamoto, I.: The crystal structure and physicochemical properties of $L$-ascorbic acid 2-glycoside. Carbohydr. Res. 232 (1992) 197-205.

7. Sheldrick, G. M.: SHELXS-97. Program for the Solution of Crystal Structures. University of Göttingen, Germany 1997.

8. Sheldrick, G. M.: SHELXL-97. Program for the Refinement of Crystal Structures. University of Göttingen, Germany 1997. 